\title{
Rasburicase in the treatment of hyperuricemia of newborns
}

\author{
Stefano Ghirardello • Gianluigi Ardissino • \\ Antonio Mastrangelo • Fabio Mosca
}

Received: 23 February 2010 /Accepted: 8 March 2010 /Published online: 8 April 2010

(C) IPNA 2010

Sirs,

The article by Hobbs and coworkers [1] provides scientific evidence for what we believe is a common clinical practice: the use of rasburicase in hyperuricemic newborns with acute kidney injury (AKI).

These authors retrospectively reviewed the charts of 7 hyperuricemic infants with AKI, successfully treated with a single dose of rasburicase $(0.17 \pm 0.04 \mathrm{mg} / \mathrm{kg})$.

During the last few years we also experienced the efficacy and safety of rasburicase in 10 AKI newborns (7 boys, 3 girls), with a mean ( $\pm \mathrm{SD}$ ) body weight of $1,226 \mathrm{~g} \mathrm{(} \pm 644.4)$, born at 29.4 weeks ( \pm 30 days) and with a mean age at administration of therapy of 20.2 days $( \pm 11.5)$. Six of them were of very low birth weight (VLBW) and 9 were premature (data not published).

After a single intravenous dose of rasburicase $(0.2 \mathrm{mg} / \mathrm{kg}$ in $30 \mathrm{~min})$ we also observed a statistically significant decrease in serum uric acid concentration of a mean level ranging from $14.5 \pm 3.6 \mathrm{mg} / \mathrm{dl}$ to $6.6 \pm 7.5 \mathrm{mg} / \mathrm{dl}(p<0.002)$. No side effects were recorded.

Hobbs and coworkers reported a significant improvement in serum creatinine and urine output on day 1 following rasburicase [1]. The authors seem to attribute these results to

S. Ghirardello $(\bowtie) \cdot$ F. Mosca

Neonatal Intensive Care Unit,

Institute of Pediatrics and Neonatology,

Fondazione IRCCS Ca' Granda Ospedale Maggiore Policlinico,

University of Milan,

via Commenda, 12 ,

Milan, Italy

e-mail: stefano.ghirardello@mangiagalli.it

G. Ardissino $\cdot$ A. Mastrangelo

Unit of Pediatric Nephrology and Dialysis and Transplantation, Department of Pediatrics,

Fondazione IRCCS Ca' Granda Ospedale Maggiore Policlinico, University of Milan,

Milan, Italy the reduction in serum uric acid as described in oncological patients with tumor lysis syndrome (TLS) [2-4]. These data are not confirmed by our study. We believe that uric acid plays a pathogenetic role in AKI secondary to TLS, while, as in the series by Hobbs et al., our patient's uric acid increased as a consequence of a reduction in urinary excretion, rather than overproduction. In fact, if we analyze each patient separately it can be speculated that the specific therapy of the underlying diseases, such as rehydration, is the main factor explaining the improvement in serum creatinine and urine output, as evidenced by the sharp decrease in blood urea nitrogen and the improvement in urine output (in patients 1, 3 , and 7) after rehydration and/or resuscitation. Moreover, in 2 cases (patients 2 and 4) serum creatinine remained significantly high during the first week after treatment.

At present, various studies have confirmed that rasburicase is effective in reducing uric acid concentration and is well tolerated $[1,4,5]$, even in the neonatal period [3] and in premature newborns, as shown by our experience.

\section{References}

1. Hobbs DJ, Steinke JM, Chung JY, Barletta GM, Bunchman TE (2010) Rasburicase improves hyperuricemia in infants with acute kidney injury. Pediatr Nephrol 25:305-309

2. Baeksgaard L, Sorensen JB (2003) Acute tumor lysis syndrome in solid tumors - a case-report and review of the literature. Cancer Chemother Pharmacol 51:187-192

3. McNutt DM, Holdsworth MT, Wong C, Hanrahan JD, Winter SS (2006) Rasburicase for the management of tumor lysis syndrome in neonates. Ann Pharmacother 40:1445-1450

4. Roncal CA, Mu W, Crocker B, Reungjui S, Ouyang X, TabahFisch I, Johnson RJ, Ejaz AA (2007) Effect of elevated serum uric acid on cisplatin-induced acute renal failure. Am J Physiol Renal Physiol 292:F116-F122

5. Ejaz AA, Mu W, Kang D-H, Roncal C, Sautin YY, Hendersen G, Tabah-Fisch I, Keller B, Beaver TM, Nakagawa T, Johnson RJ (2007) Could uric acid have a role in acute renal failure? Clin J Am Soc Nephrol 2:16-21 